\title{
Inter- and Intragrain Inhomogeneity in 2D Perovskite Thin Films Revealed by Relative Grain Orientation Imaging Using Low-Frequency Polarized Raman Microspectroscopy
}

\author{
Shogo Toda,${ }^{\dagger}$ Naoya Yanagita,${ }^{\dagger}$ Efat Jokar, ${ }^{\dagger}, \S$ Eric Wei-Guang Diau, $,{ }^{\dagger}, \S$ and \\ Shinsuke Shigeto*,†
}

†Department of Chemistry, Graduate School of Science and Technology, Kwansei Gakuin University, Hyogo 669-1337, Japan

\$Department of Applied Chemistry and Institute of Molecular Science, National Chiao Tung University, Hsinchu 30010, Taiwan

${ }^{\S}$ Center for Emergent Functional Matter Science, National Chiao Tung University, Hsinchu 30010, Taiwan

*Corresponding author

E-mail: shigeto@kwansei.ac.jp

\section{Table of Contents}

$\begin{array}{lll}\text { Experimental Section } & \ldots \ldots \ldots \ldots & \text { S2 } \\ \text { Table S1 } & \ldots \ldots \ldots \ldots & \text { S4 } \\ \text { Figure S1 } & \ldots \ldots \ldots \ldots & \text { S4 } \\ \text { Figure S2 } & \ldots \ldots \ldots \ldots & \text { S4 } \\ \text { Figure S3 } & \ldots \ldots \ldots \ldots & \text { S5 } \\ \text { Figure S4 } & \ldots \ldots \ldots \ldots & \text { S5 } \\ \text { References } & \ldots \ldots \ldots \ldots & \text { S6 }\end{array}$




\section{Experimental Section}

\section{Materials}

Synthesis of $\mathbf{B A}_{2} \mathrm{PbI}_{4}$. The synthesis of $\left(\mathrm{C}_{4} \mathrm{H}_{9} \mathrm{NH}_{3}\right)_{2} \mathrm{PbI}_{4}\left(\mathrm{BA}_{2} \mathrm{PbI}_{4}\right) 2 \mathrm{D}$ perovskite crystals was performed based on the procedures reported elsewhere. ${ }^{1,2}$ Briefly, $10 \mathrm{mmol} \mathrm{PbO}$ powder (Alfa Aesar) was dissolved in a mixture of $10 \mathrm{~mL} \mathrm{HI} \mathrm{(57 \% ,} \mathrm{Alfa} \mathrm{Aesar)} \mathrm{and} 1.7 \mathrm{~mL} \mathrm{H} \mathrm{PO}_{2}(50 \%$, aqueous, Alfa Aesar) at room temperature. The clear-yellow solution was boiled and kept boiling about $5 \mathrm{~min}$. $N$-Butylamine ( $924 \mu \mathrm{L}, 10 \mathrm{mmol}$, Sigma Aldrich) was neutralized with excess $\mathrm{HI}(5 \mathrm{~mL})$ in another vial and was dropwise added to the boiling $\mathrm{PbI}_{2}$ solution. After the solution became clear, stirring and heating the solution was discontinued, and the solution was left to cool down to room temperature. The obtained orange plate-shape crystals were collected by suction filtration and dried under low pressure at room temperature for a few days.

Film fabrication. The synthesized $\mathrm{BA}_{2} \mathrm{PbI}_{4}$ was dissolved in dimethylformamide (DMF, anhydrous, FUJIFILM Wako) to yield a $45 \mathrm{wt} \%$ solution of $\mathrm{BA}_{2} \mathrm{PbI}_{4}$. The solution was heated to $70{ }^{\circ} \mathrm{C}$ with stirring for $3 \mathrm{~h}$, followed by filtration with a $0.45 \mu \mathrm{m}$ polytetrafluoroethylene (PTFE) filter. The resulting solution $(40 \mu \mathrm{L})$ was spun onto an $\mathrm{O}_{2}$ plasma-treated glass cover slip at $4000 \mathrm{rpm}$ for $15 \mathrm{~s}$. The $\mathrm{BA}_{2} \mathrm{PbI}_{4}$ thin film was then annealed at $100{ }^{\circ} \mathrm{C}$ on a petri dish for $10 \mathrm{~min}$. After heating, $20 \mu \mathrm{L}$ of DMF was dropped around the perovskite film, generating DMF vapor inside the covered petri dish. The perovskite film exposed to the DMF vapor was maintained at $100{ }^{\circ} \mathrm{C}$ for another $10 \mathrm{~min}$, during which a growth of larger grains of the perovskite was promoted (solvent annealing ${ }^{3}$ ). Finally, the film was left for 1 min without the cover so as to completely remove DMF. All these procedures were performed in a nitrogenfilled glove box.

\section{Spectroscopic measurements}

Confocal LFPR microspectroscopy. The confocal Raman microspectrometer used in this work is basically the same as that already reported elsewhere. ${ }^{4}$ The $632.8 \mathrm{~nm}$ output of a HeNe laser was used as the excitation light. After passing through a half-wave plate and a GlanThompson polarizer for polarization control, the laser beam was introduced to an inverted microscope (Nikon, TE2000-U, customized) using a volume Bragg grating (VBG) notch filter (OptiGrate, $\mathrm{OD}=3$ ) and a dichroic mirror, and focused onto the sample by a $100 \times, \mathrm{NA}=1.45$, oil-immersion objective (Nikon, Plan Apo $\lambda$ ). The laser power at the sample point was adjusted to be $0.4 \mathrm{~mW}$. The sample was set on a three-axis piezoelectric nanopositioner (Mad City Labs, Nano-LP100) mounted on the microscope stage. Backscattered light was collected by the same objective. The well-collimated scattered light was passed through two more VBGs, which eliminated Rayleigh scattering very efficiently with an extremely narrow band width. ${ }^{5}$ Owing to these VBGs, ultralow-frequency Raman spectra down to $\sim 10 \mathrm{~cm}^{-1}$ were successfully observed. The scattered light was then focused onto a $100-\mu \mathrm{m}$ pinhole with a pair of lenses to achieve confocal detection. Another Glan-Thompson polarizer was used as an analyzer. The transmitted light was analyzed by an imaging spectrometer (SOL Instruments, MS3501i) and 
detected with an electron-multiplying charge-coupled device (EMCCD) detector (Andor Technology, Newton). A $600 \mathrm{~mm}^{-1}$ grating was used when measuring the low-frequency and high-frequency regions simultaneously (see Figure 2b), whereas a $1800 \mathrm{~mm}^{-1}$ grating was used when measuring only the low-frequency or high-frequency region with a higher spectral resolution. The spectral resolution of our spectrometer was $4 \mathrm{~cm}^{-1}$ for the $600 \mathrm{~mm}^{-1}$ grating and $2 \mathrm{~cm}^{-1}$ for the $1800 \mathrm{~mm}^{-1}$ grating.

In LFPR imaging experiments, the sample was translated with the piezoelectric stage in a raster manner. $20 \times 20 \mu \mathrm{m}^{2}$ and $60 \times 60 \mu \mathrm{m}^{2}$ area were scanned with steps of 0.5 and $1.5 \mu \mathrm{m}$, respectively. Therefore, in both cases, a total of $41 \times 41=1681$ Raman spectra were acquired. The exposure time at each point was $1 \mathrm{~s}$. The step size was larger than the estimated spatial resolution in lateral $(X Y)$ direction $\sim 0.4 \mu \mathrm{m}$. The spatial resolution in axial $(Z)$ direction was estimated to be $\sim 4 \mu \mathrm{m}$.

Visible absorption spectroscopy. The visible absorption spectrum of a $\mathrm{BA}_{2} \mathrm{PbI}_{4}$ thin film fabricated on a plasma-treated glass cover slip was measured with a spectrophotometer (JASCO, $\mathrm{V}-750$ ). An integrating sphere was used to remove the effects of scattering and reflection caused by the sample. The spectral resolution was set to be $2 \mathrm{~nm}$.

\section{Spectral analysis}

First of all, LFPR imaging data were subjected to noise reduction using singular value decomposition (SVD). ${ }^{6-8}$ The largest 10 singular components were retained to reconstruct denoised data. All of the other singular components were discarded as noises. A baseline in the low-frequency region was fitted to a quadratic function for $Z(X X) Z$ and $Z(Y Y) Z$, and to a linear function for $Z(X Y) Z$ and $Z(Y X) Z$. This baseline correction and curve fitting with Gaussian functions and their second derivatives (Figure 4a) were performed on Igor Pro 8 (WaveMetrics).

For hierarchical cluster analysis (HCA), all SVD-denoised Raman spectra were vectornormalized. HCA was performed on R software (version 3.5.3). Euclidean distances were used as a distance measure, and Ward's algorithm was used to partition Raman spectra into clusters and generate a dendrogram. 
Table S1. Peak positions and band widths (full width at half-maximum) of the six Gaussian bands used to fit the LFPR data.

\begin{tabular}{|c|c|c|}
\hline Band \# & Peak position $/ \mathrm{cm}^{-1}$ & Band width $/ \mathrm{cm}^{-1}$ \\
\hline 1 & 18.0 & 6.5 \\
\hline 2 & 20.0 & 5.2 \\
\hline 3 & 25.9 & 8.1 \\
\hline 4 & 32.7 & 11.5 \\
\hline 5 & 43.2 & 10.1 \\
\hline 6 & 46.2 & 9.8 \\
\hline
\end{tabular}

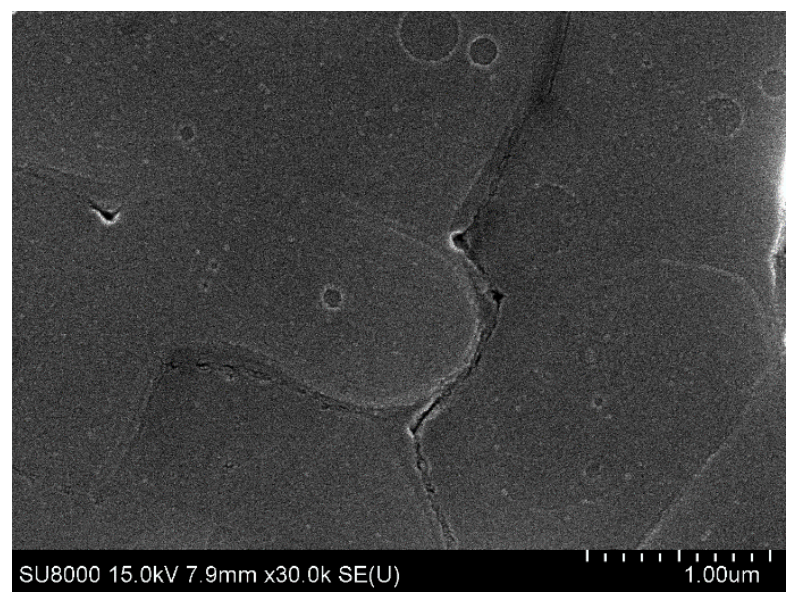

Figure S1. Scanning electron microscopy (SEM) image of a $\mathrm{BA}_{2} \mathrm{PbI}_{4}$ thin film. Note that this image was not taken from the same film as those in Figures $2 \mathrm{a}$ and $3 \mathrm{a}$ and is for reference only.

a

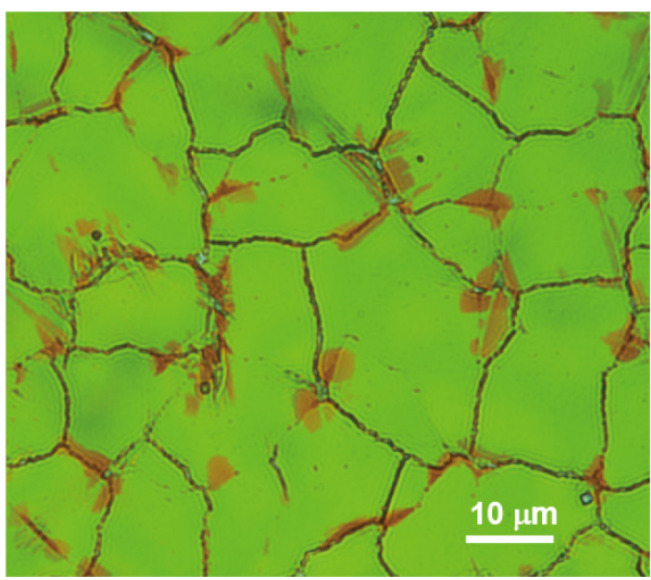

b

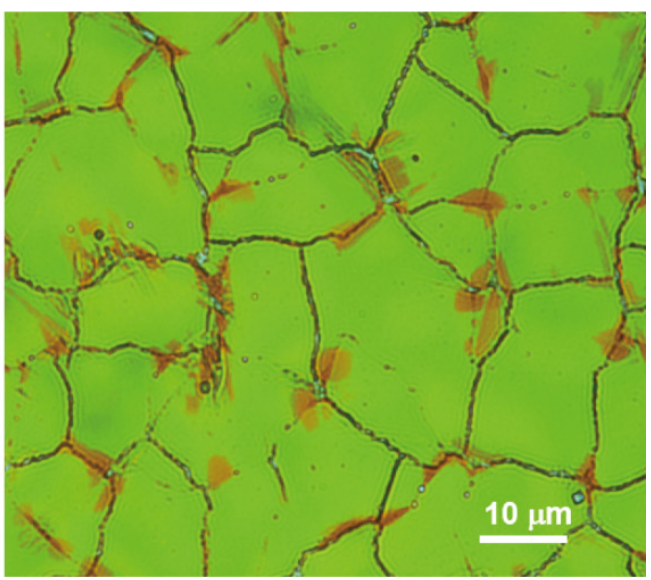

Figure S2. Comparison of the optical micrographs of the $\mathrm{BA}_{2} \mathrm{PbI}_{4}$ thin film captured before (a) and after (b) the four sets of LFPR imaging experiments, which took approximately $2 \mathrm{~h}$. 


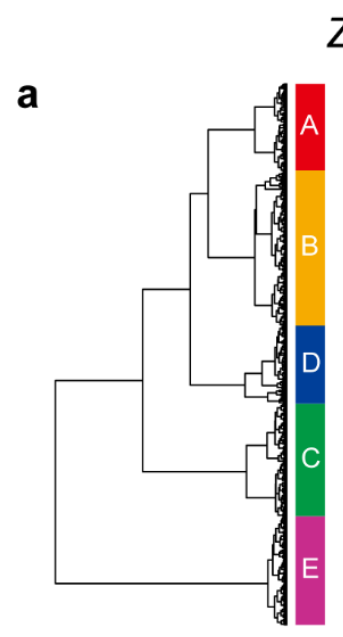

$Z(X X) Z$

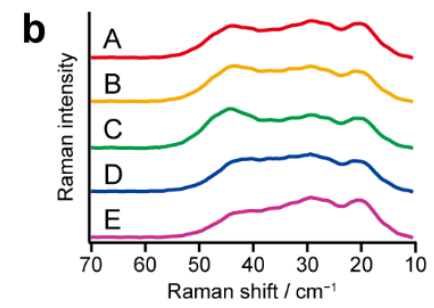

C

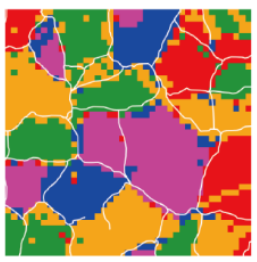

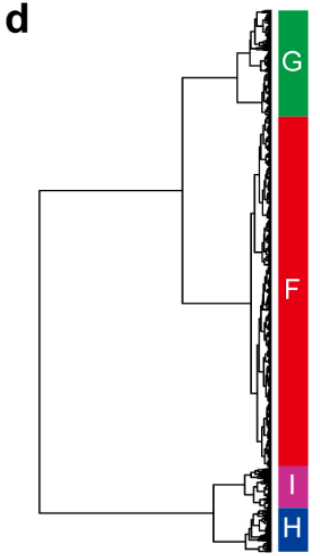

$Z(X Y) Z$
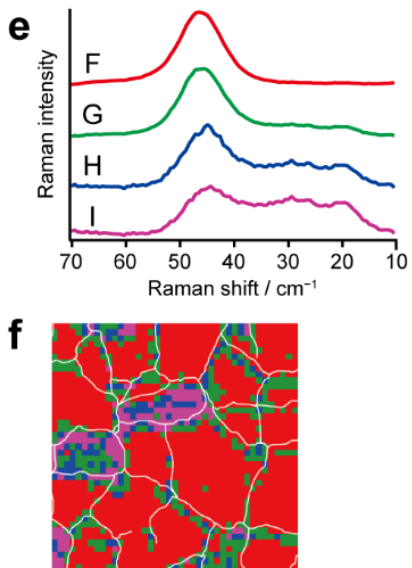

Figure S3. (a-c) HCA results for the $Z(X X) Z$ LFPR imaging data of the $\mathrm{BA}_{2} \mathrm{PbI}_{4}$ film. Dendrogram (a) showing a classification of the data into five clusters A-E; mean Raman spectra (b) and spatial distributions (c) of clusters A-E. (d-f) HCA results for the $Z(X Y) Z$ LFPR imaging data of the $\mathrm{BA}_{2} \mathrm{PbI}_{4}$ film. Dendrogram (d) showing a classification of the data into four clusters F-I; mean Raman spectra (e) and spatial distributions (f) of clusters F-I.
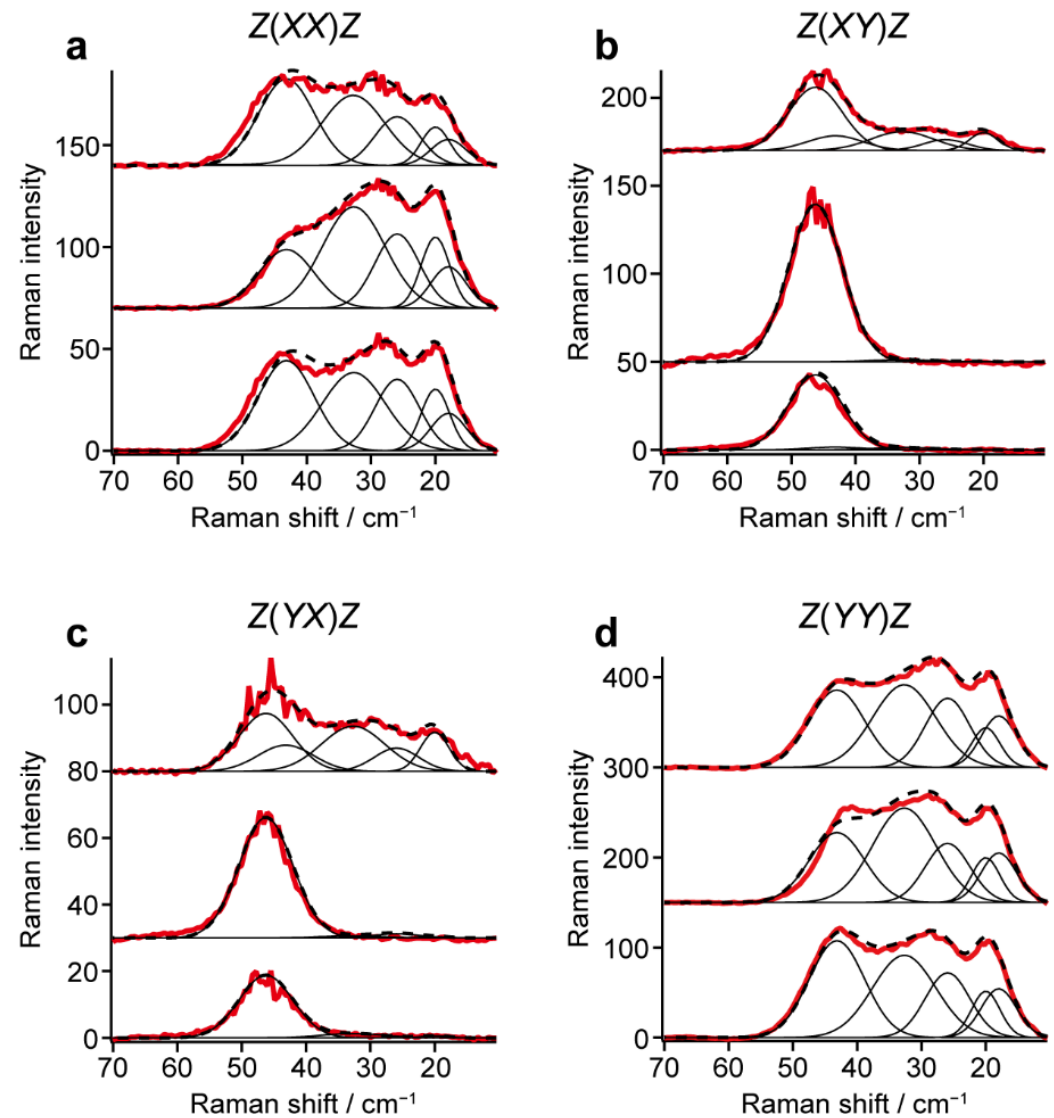

Figure S4. Representative results of the fitting with five Gaussian bands for the $Z(X X) Z$ (a), $Z(X Y) Z(\mathrm{~b}), Z(Y X) Z(\mathrm{c})$, and $Z(Y Y) Z$ (d) polarization configurations. Red solid lines, observed Raman spectra; dashed black lines, best fits; solid black lines, five Gaussian bands. 


\section{References}

(1) Stoumpos, C. C.; Cao, D. H.; Clark, D. J.; Young, J.; Rondinelli, J. M.; Jang, J. I.; Hupp, J. T.; Kanatzidis, M. G., Ruddlesden-Popper Hybrid Lead Iodide Perovskite 2D Homologous Semiconductors. Chem. Mater. 2016, 28, 2852-2867.

(2) Kattoor, V.; Awasthi, K.; Jokar, E.; Diau, E. W.-G.; Ohta, N., Integral Method Analysis of Electroabsorption Spectra and Electrophotoluminescence Study of $\left(\mathrm{C}_{4} \mathrm{H}_{9} \mathrm{NH}_{3}\right)_{2} \mathrm{PbI}_{4}$ Organic-Inorganic Quantum Well. J. Phys. Chem. C 2018, 122, 26623-26634.

(3) Liu, J.; Gao, C.; He, X.; Ye, Q.; Ouyang, L.; Zhuang, D.; Liao, C.; Mei, J.; Lau, W., Improved Crystallization of Perovskite Films by Optimized Solvent Annealing for High Efficiency Solar Cell. ACS Appl. Mater. Interfaces 2015, 7, 24008-24015.

(4) Matsuda, A.; Sakaguchi, N.; Shigeto, S., Can Cells Maintain Their Bioactivity in Ionic Liquids? A Novel Single-Cell Assessment by Raman Microspectroscopy. J. Raman. Spectrosc. 2019, 50, 768-777.

(5) Chang, C.-F.; Okajima, H.; Hamaguchi, H.; Shigeto, S., Imaging Molecular Crystal Polymorphs and Their Polycrystalline Microstructures in Situ by Ultralow-Frequency Raman Spectroscopy. Chem. Commun. 2014, 50, 12973-12976.

(6) Uzunbajakava, N.; Lenferink, A.; Kraan, Y.; Volokhina, E.; Vrensen, G.; Greve, J.; Otto, C., Nonresonant Confocal Raman Imaging of DNA and Protein Distribution in Apoptotic Cells. Biophys. J. 2003, 84, 3968-3981.

(7) van Manen, H.-J.; Kraan, Y. M.; Roos, D.; Otto, C., Intracellular Chemical Imaging of Heme-Containing Enzymes Involved in Innate Immunity Using Resonance Raman Microscopy. J. Phys. Chem. B 2004, 108, 18762-18771.

(8) Huang, C.-K.; Hamaguchi, H.; Shigeto, S., In Vivo Multimode Raman Imaging Reveals Concerted Molecular Composition and Distribution Changes During Yeast Cell Cycle. Chem. Commun. 2011, 47, 9423-9425. 